\title{
Pulmonary Infection with an Interferon- $\gamma$-Producing Cryptococcus neoformans Strain Results in Classical Macrophage Activation and Protection
}

\author{
Sarah E. Hardison, ${ }^{\star \dagger}$ Sailatha Ravi, ${ }^{\star \dagger}$ \\ Karen L. Wozniak, ${ }^{* \dagger}$ Mattie L. Young, ${ }^{* \dagger}$ \\ Michal A. Olszewski, ${ }^{\ddagger \S}$ and Floyd L. Wormley Jr. ${ }^{* \dagger}$ \\ From the Department of Biology* and The South Texas Center for \\ Emerging Infectious Diseases, ${ }^{\dagger}$ The University of Texas at San \\ Antonio, San Antonio, Texas; the VA Ann Arbor Health System, \\ Ann Arbor Michigan; and the Division of Pulmonary and \\ Critical Care Medicine, Department of Internal Medicine, \\ University of Michigan Health System, Ann Arbor, Michigan
}

\begin{abstract}
Alternative macrophage activation is associated with exacerbated disease in murine models of pulmonary cryptococcosis. The present study evaluated the efficacy of interferon- $\gamma$ transgene expression by Cryptococcus neoformans strain $\mathrm{H} 99 \gamma$ in abrogating alternative macrophage activation in infected mice. Macrophage recruitment into the lungs of mice after infection with $C$. neoformans strain $\mathrm{H} 99 \gamma$ was comparable with that observed in mice challenged with wild-type $C$. neoformans. However, pulmonary infection in mice with $C$. neoformans strain $\mathrm{H} 99 \gamma$ was associated with reduced pulmonary fungal burden, increased pulmonary Th1-type and interleukin-17 cytokine production, and classical macrophage activation as evidenced by increased inducible nitric oxide synthase expression, histological evidence of enhanced macrophage fungicidal activity, and resolution of inflammation. In contrast, progressive pulmonary infection, enhanced Th2-type cytokine production, and the induction of alternatively activated macrophages expressing arginase-1, found in inflammatory zone 1, Ym1, and macrophage mannose receptor were observed in the lungs of mice infected with wild-type $C$. neoformans. These alternatively activated macrophages were also shown to harbor highly encapsulated, replicating cryptococci. Our results demonstrate that pulmonary infection with $C$. neoformans strain $\mathbf{H} 99 \gamma$ results in the induction of classically activated macrophages and promotes fungal clearance. These studies indicate that phenotype, as opposed to quantity, of infiltrating macrophages cor-
\end{abstract}

relates with protection against pulmonary $C$. neoformans infection. (Am J Pathol 2010, 176:774-785; DOI: 10.2353/ajpath.2010.090634)

Cryptococcus neoformans is an opportunistic fungal pathogen and frequent cause of life-threatening infection in individuals with suppressed cell-mediated immunity. ${ }^{1}$ C. neoformans is the most common mycological agent of morbidity and mortality in patients with AIDS with acute mortality rates ranging between 10 and $25 \%$ in developed countries worldwide. ${ }^{2}$ Infection is initiated after the inhalation of desiccated basidiospores or yeast into lung alveoli, resulting in asymptomatic disease or mild bronchopneumonia in immunocompetent individuals. ${ }^{1}$ However, bronchial infection is severe in immunocompromised patients and often leads to dissemination, resulting in severe meningoencephalitis. As inhalation is the principal route of entry for $C$. neoformans, clearance from the lungs is largely dependent on the ability of resident alveolar macrophages to degrade the yeast cells, thereby preventing dissemination.

Experimental murine models of pulmonary C. neoformans infection suggest that resolution of infection is associated with the induction of Th1-type cytokine responses characterized by the production of interleukin (IL)-2, IL-12, tumor necrosis factor- $\alpha$, and interferon (IFN)- $\gamma^{3-11}$ These cytokines, in turn, induce lymphocyte and phagocyte recruitment and activation of anticryptococcal delayed-type hypersensitivity responses. In contrast, uncontrolled fungal growth and exacerbation of pulmonary cryptococcosis is associated with Th2-type

Supported by research grants R01-AI071752-03 from the National Institute of Allergy and Infectious Diseases (NIAID) of the National Institutes of Health (NIH) (F.L.W.) and a Merit Review grant from the Department of Veterans Affairs (M.A.O.). The content is solely the responsibility of the authors and does not necessarily represent the official views of the NIAID $\mathrm{NIH}$.

\section{Accepted for publication October 16, 2009.}

Address reprint requests to Floyd L. Wormley Jr, Ph.D., Department of Biology, The University of Texas at San Antonio, One UTSA Circle, San Antonio, TX 78249-0062. E-mail: floyd.wormley@utsa.edu. 
cytokine responses and the generation of alternatively activated macrophages (aaMac). ${ }^{4,11,12}$ Specifically, aaMac are induced in high IL-4/IL-13 environments and are thought to contribute to pulmonary pathology by a variety of means. ${ }^{12-15}$ First, aaMac up-regulate genes that increase cryptococcal persistence within macrophages, including arginase-1 (Arg1) and the macrophage mannose receptor (CD206). Up-regulation of Arg1 decreases synthesis of fungicidal nitric oxide by competing with inducible nitric oxide synthase (iNOS) for the substrate L-arginine. ${ }^{16}$ Increased surface expression of CD206 results in increased phagocytosis but is accompanied by decreased intracellular killing and TNF- $\alpha$ production. ${ }^{17-20}$ Second, aaMac up-regulate proteins implicated in pulmonary pathology, such as chitinase family proteins Ym1, Ym2, and AMCase as well as found in inflammatory zone 1 (FIZZ1) protein. ${ }^{4,12,13}$ Pulmonary C. neoformans infection in C57BL/6 mice is characterized by enhanced alternative macrophage activation and disease progression. ${ }^{4,13}$ Interestingly, C57BL/6 mice deficient in IFN- $\gamma$ develop augmented Th2-type cytokine production and the induction of aaMac during pulmonary $C$. neoformans infection. ${ }^{4}$ Moreover, IL-13 promotes aaMac differentiation, Th2-type cytokine responses, and allergic inflammation during experimental pulmonary cryptococcosis in mice. ${ }^{11}$ Thus, alternative macrophage activation has a clear role in promoting progressive cryptococcal disease. ${ }^{4,15,21}$

Experimental pulmonary infection with the wild-type $C$. neoformans strain H99 results in fatal outcomes associated with overexuberant Th2-type cytokine responses in a variety of mouse models. ${ }^{12,22}$ In contrast, experimental pulmonary infection of BALB/c mice with a $C$. neoformans strain $\mathrm{H} 99$ engineered to produce murine IFN- $\gamma$ (designated $\mathrm{H} 99 \gamma$ ) results in the induction of Th1-type cytokines as well as a significant influx of T cells, granulocytes, and antigen-presenting cells into the lungs. ${ }^{23}$ However, the effect of IFN- $\gamma$ transgene expression by $C$. neoformans strain $\mathrm{H} 99 \gamma$ on the macrophage activation profile in infected lungs remains unknown. The objective of these studies was to determine the activation phenotype of macrophages elicited in response to pulmonary infection with C. neoformans strain $\mathrm{H} 99 \gamma$ compared with that observed in mice infected with the wild-type $C$. neoformans.

\section{Materials and Methods}

\section{Mice}

Female BALB/c $\left(\mathrm{H}-2^{\mathrm{d}}\right)$ mice, 4 to 6 weeks of age (National Cancer Institute/Charles River Laboratories, Wilmington, MA), were used in these studies. Mice were housed at The University of Texas at San Antonio Small Animal Laboratory vivarium and handled according to guidelines approved by the institutional animal care and use committee.

\section{Strains and Media}

C. neoformans strains H99 (serotype A, Mat $\alpha$ ) and H99 (an interferon- $\gamma$ producing C. neoformans strain derived from $\mathrm{H}^{23}{ }^{23}$ ) were recovered from $15 \%$ glycerol stocks stored at $-80^{\circ} \mathrm{C}$ before use in the experiments described herein. The strains were maintained on yeast extractpeptone-dextrose (YPD) medium (BD Diagnostic Systems, Sparks, MD). Yeast cells were grown for 14 to 16 hours at $30^{\circ} \mathrm{C}$ with shaking in YPD broth (BD Diagnostic Systems), collected by centrifugation, and washed three times with sterile PBS, and viable yeast was quantified using trypan blue dye exclusion in a hemacytometer.

\section{Murine Model}

Pulmonary C. neoformans infections were initiated by nasal inhalation as described previously. ${ }^{24,25}$ In brief, $\mathrm{BALB} / \mathrm{c}$ mice were anesthetized with $2 \%$ isoflurane using a rodent anesthesia device (Eagle Eye Anesthesia, Jacksonville, FL) and then given a yeast inoculum of $1 \times 10^{4}$ colony forming units (CFU) of $C$. neoformans strains H99 or $\mathrm{H} 99 \gamma$ in $50 \mu \mathrm{l}$ of sterile PBS pipetted directly into the nares. The inocula used for nasal inhalation were verified by quantitative culture on YPD agar. The mice were fed ad libitum and monitored by inspection twice daily. Mice were euthanized on predetermined days after inoculation, and lung tissues were excised using aseptic technique, homogenized in $1 \mathrm{ml}$ of sterile PBS, and cultured by 1:10 dilutions on YPD agar supplemented with chloramphenicol (Mediatech, Inc., Herndon, VA). CFU were enumerated after incubation at $30^{\circ} \mathrm{C}$ for 48 hours.

\section{Pulmonary Leukocyte Isolation}

Lungs were excised on days 3,7 , and 14 postinoculation and digested enzymatically at $37^{\circ} \mathrm{C}$ for 30 minutes in 10 $\mathrm{ml}$ of digestion buffer (RPMl 1640 and $1 \mathrm{mg} / \mathrm{ml}$ of collagenase type IV [Sigma Chemical Co., St. Louis, MO]) with intermittent (every 10 minutes) stomacher homogenizations. The enzymatically digested tissues were then successively filtered through sterile nylon filters of various pore sizes (70 and $40 \mu \mathrm{m}$ ) (BD Biosciences, San Jose, $\mathrm{CA}$ ) and washed with sterile Hanks' balanced salt solution to enrich for leukocytes. Erythrocytes were lysed by incubation in $\mathrm{NH}_{4} \mathrm{Cl}$ buffer $\left(0.859 \% \mathrm{NH}_{4} \mathrm{Cl}, 0.1 \% \mathrm{KHCO}\right.$, and $0.0372 \% \mathrm{Na}_{2}$ EDTA [pH 7.4], Sigma Chemical Co.) for 3 minutes on ice followed by a 10 -fold excess of PBS. The resulting leukocyte population was then collected by centrifugation $(800 \times g$ for 5 minutes, washed twice with sterile PBS, resuspended in sterile PBS containing $2 \%$ heat-inactivated fetal bovine serum (fluorescence-activated cell sorting [FACS] buffer) and enumerated in a hemacytometer using trypan blue dye exclusion. For gene expression analysis, the leukocyte population was enriched for macrophages by positive selection using magnetic beads labeled with CD11b antibody according to the manufacturer's recommendations (Miltenyi Biotec, Auburn, CA).

\section{Antibodies}

For flow cytometry experiments, rat anti-mouse CD16/ CD32 (Fc Block) (BD Pharmingen, San Diego, CA), rat 
anti-mouse CD45 (BD Pharmingen) conjugated to phycoerythrin (PE), rat anti-mouse F4/80 conjugated to allophycocyanin (Caltag Laboratories, Burlingame, CA) or to phycoerythrin (eBioscience, San Diego, CA), rat antimouse IL-4 conjugated to PE-Cy7 (eBioscience), and IL-13 conjugated to Alexa Fluor 647 (eBioscience) were used. For immunohistochemistry experiments, rabbit anti-mouse Arg1 (Santa Cruz Biotechnology, Inc., Santa Cruz, CA), rat anti-mouse CD206 (macrophage mannose receptor) (AbD Serotec, Raleigh, NC), rat anti-mouse Ym1 (R\&D Systems, Minneapolis, MN), rat anti-mouse F4/80 (AbD Serotec), and rabbit anti-mouse iNOS (Axxora, LLC, San Diego, CA) were used. Primary antibodies were detected using appropriate fluorescence isothiocyanate-conjugated goat anti-rat IgG (Jackson ImmunoResearch Laboratories, Inc., West Grove, PA) or goat anti-rabbit IgG (Jackson ImmunoResearch Laboratories, Inc.) secondary antibodies.

\section{Flow Cytometry}

Standard methodology was used for the direct and indirect immunofluorescence of pulmonary leukocytes. In brief, in 96-well U-bottom plates $1 \times 10^{6}$ leukocyteenriched lung cells were incubated with Fc Block in $50 \mu$ l of PBS for 5 minutes to block nonspecific binding of antibodies to cellular Fc receptors. Subsequently, optimal concentrations of CD45 and F4/80 antibodies were added to allow for dual staining in $50 \mu$ l of FACS buffer. After 30 minutes of incubation on ice, the cells were washed three times with FACS buffer and then were fixed in $200 \mu \mathrm{l}$ of $2 \%$ ultrapure formaldehyde (Polysciences, Inc., Warrington, PA). Cells were incubated with either FACS buffer alone or single fluorochrome-conjugated antibodies to determine positive staining and spillover/compensation calculations, and background fluorescence was determined by the flow cytometer.

Leukocytes were also stained for intracellular cytokine expression. Cells were initially labeled with anti-F4/80 antibodies as outlined above and fixed with $100 \mu \mathrm{l}$ of $2 \%$ ultrapure formaldehyde (Polysciences, Inc.) at room temperature for 10 minutes. The cells were then permeabilized using $0.1 \%$ saponin (Sigma Chemical Co.) for 10 minutes at room temperature, and optimal concentrations of anti-IL-4 and IL-13 antibodies were added. Cells were incubated with intracellular antibodies at $4^{\circ} \mathrm{C}$ for $30 \mathrm{~min}$ utes. After staining, the cells were washed three times with $0.1 \%$ saponin and then fixed in $200 \mu \mathrm{l}$ of $2 \%$ ultrapure formaldehyde. Samples were analyzed using the software provided with a BD FACSArray flow cytometer (BD Pharmingen). Dead cells were excluded on the basis of forward angle and $90^{\circ}$ light scatter. For data analyses, 30,000 events (cells) were evaluated, and the absolute number of $\mathrm{CD} 45^{+} / \mathrm{F} 4 / 80^{+}$or the percentage of $\mathrm{F} 4 / 80^{+} /$ $\mathrm{IL}-4$, and $\mathrm{F} 4 / 80^{+} / \mathrm{IL}-13$ cells was calculated.

\section{Real-Time PCR}

Total RNA was isolated from purified CD11 $\mathrm{b}^{+}$cells using TRIzol reagent (Invitrogen, Carlsbad, CA) and then
DNase (Invitrogen)-treated to remove possible traces of contaminating DNA according to the manufacturer's instructions. First-strand cDNA was synthesized from $1 \mu \mathrm{g}$ of total RNA using the oligo(dT) primer, and reagents supplied in the SuperScript III RT Kit (Invitrogen) according to the manufacturer's instructions. The cDNA was used as a template for analysis by real-time PCR using the TaqMan Gene Expression Assay (Applied Biosystems, Foster City, CA) according to manufacturer's instructions. All real-time PCR reactions were performed using the 7300 Real-Time PCR System (Applied Biosystems). For each real-time PCR reaction, a master mix was prepared on ice with TaqMan Gene Expression Assays specific for iNOS, IFN- $\gamma$, Ym1, FIZZ1, Arg1, IL-4, IL-13, IL-17, and CD206 (Applied Biosystems). TaqMan rodent glyceraldehyde-3-phosphate dehydrogenase (GAPDH) (Applied Biosystems) was used as an internal control. The thermal cycling parameters contained an initial denaturing cycle of $95^{\circ} \mathrm{C}$ for 10 minutes followed by 40 cycles of $95^{\circ} \mathrm{C}$ for 15 seconds and $60^{\circ} \mathrm{C}$ for 60 seconds. Results of the real-time PCR data were derived using the comparative $\mathrm{Ct}$ method as described previously ${ }^{26-28}$ to detect relative gene expression. The parameter $\mathrm{Ct}$ is defined as the cycle number at which the amplification plot passes a fixed threshold above baseline. Each reaction was run in triplicate in separate wells and normalized to a control endogenous gene, GAPDH. The following formula was used to quantify the fold differential expression of a specific gene in a macrophage-enriched population after pulmonary inoculation with $C$. neoformans strain $\mathrm{H} 99 \gamma$ compared with wild-type infected mice: $2^{-\Delta \Delta \mathrm{Ct}}$, where $\Delta \Delta \mathrm{Ct}=[\mathrm{Ct} \mathrm{H} 99 \gamma$ sample $-\mathrm{Ct}$ GAPDH of H99 $\gamma$ sample] - [Ct H99 sample - Ct GAPDH of H99 sample]. Ct represents the mean $\mathrm{Ct}$ value of each sample in triplicate. Therefore, the result represents the fold increase or decrease in the expression of the gene in question after infection with $\mathrm{C}$. neoformans strain $\mathrm{H} 99 \gamma$ compared with wild-type $C$. neoformans.

\section{Immunohistochemistry and Histology}

Mice were continuously sedated at predetermined time points using $2 \%$ isoflurane as stated previously. Lungs were perfused with sterile PBS by transcardial perfusion through the right ventricle. The pericardium and trachea were exposed by dissection and an incision was made in the trachea for the insertion of a sterile flexible cannula attached to a 3-ml syringe. The lungs were then slowly inflated with 0.5 to $0.7 \mathrm{ml}$ of a Tissue-Tek optimal cutting temperature (OCT) compound (Sakura Finetek, Torrance, $\mathrm{CA}) / 2 \mathrm{~mol} / \mathrm{L}$ sucrose $(1: 1, \mathrm{v} / \mathrm{v})$ solution. The lungs were then excised and immediately preserved in cryomolds containing OCT medium on dry ice and stored at $-80^{\circ} \mathrm{C}$ until use.

Serial frozen tissue sections were cut at a thickness of $10 \mu \mathrm{m}$ and fixed at $-20^{\circ} \mathrm{C}$ in acetone for 10 minutes. Tissue sections were then stained using H\&E (The University of Texas Health Sciences Center at San Antonio Histology and Immunohistochemistry Laboratory) or further processed for immunohistochemical analysis to 
visualize the leukocyte infiltration. Tissue sections were placed in cold $\left(-20^{\circ} \mathrm{C}\right) 70 \%$ ethanol for 5 minutes and washed in PBS for 3 minutes. Nonspecific binding was inhibited by blocking for 30 minutes at room temperature with serum from the same species from which the fluorochrome-conjugated antibodies were derived. Tissue sections were incubated overnight at $4^{\circ} \mathrm{C}$ with primary antibodies diluted in species-specific serum (3\% in PBS) at preoptimized concentrations. Subsequently, the sections were washed seven times in Tris- $\mathrm{NaCl}$ Tween 20 (TNT) buffer solution for 3 minutes each time. Sections were then incubated with secondary antibodies for 30 minutes at room temperature. Slides were then washed seven times in TNT buffer for 3 minutes each time, one time in PBS containing $1 \%$ Triton $X$ to minimize background fluorescence ( 3 minutes) and a final wash in TNT buffer (3 minutes). Sections were then mounted with FluorSave reagent (Calbiochem, La Jolla, CA) containing $0.3 \mu \mathrm{mol} / \mathrm{L}$ 4',6'-diamidino-2-phenylindole dilactate (DAPI) (Molecular Probes, Eugene, OR). Fluorescence was visualized with a Leica DMR epifluorescence microscope (Leica Microsystems, Wetzlar, Germany). Images were acquired using a cooled SPOT RT charge-coupled device camera (Diagnostic Instruments Inc., Sterling Heights, MI).

\section{Cytokine Analysis}

Cytokine production in lung tissues was analyzed using the Bio-Plex Protein Array System (Luminex-based technology) (Bio-Rad Laboratories, Hercules, CA). In brief, lung tissue was excised and homogenized in ice-cold sterile PBS ( $1 \mathrm{ml})$. An aliquot ( $50 \mu \mathrm{l})$ was taken to quantify the pulmonary fungal burden, and an anti-protease buffer solution (1 ml) containing PBS, protease inhibitors (inhibiting cysteine, serine, and other metalloproteinases) and 0.05\% Triton $X-100$ was added to the homogenate that was then clarified by centrifugation $(800 \times g)$ for 5 minutes. Pulmonary homogenates were assayed undiluted for cytokine production using the Bio-Plex Protein Array System.

\section{Statistical Analysis}

An unpaired Student's t-test (two-tailed) using GraphPad Prism (version 5.00 for Windows, GraphPad Software, Inc., San Diego, CA) was used to detect statistically significant differences. Statistically significant differences were defined as $P<0.05$.

\section{Results}

\section{Effect of IFN- $\gamma$ Transgene Expression by C. neoformans Strain H99y on Pulmonary Fungal Burden and Macrophage Recruitment}

Previous results in our laboratory have demonstrated that BALB/c mice given an experimental pulmonary infection with $C$. neoformans strain $\mathrm{H} 99 \gamma$, a transgenic strain expressing murine IFN- $\gamma$, were able to resolve the acute

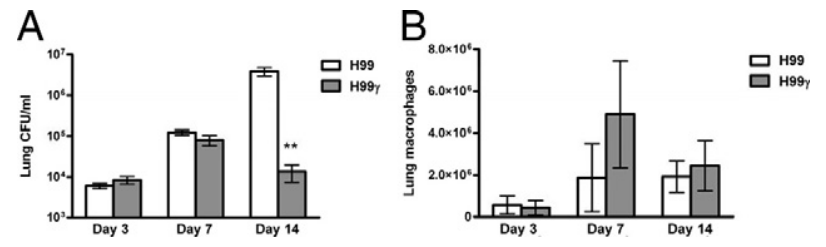

Figure 1. Resolution of pulmonary infection with $C$. neoformans strain $\mathrm{H} 99 \gamma$ is not associated with increased macrophage recruitment. BALB/c mice received an intranasal inoculum of $1 \times 10^{4} \mathrm{CFU}$ of $C$. neoformans strains $\mathrm{H} 99$ or $\mathrm{H} 99 \gamma$ in $50 \mu \mathrm{l}$ of sterile PBS. The lungs from each group of mice were excised at days 3, 7, and 14 after secondary inoculation, and the fungal burden was quantified (A). Alternatively, pulmonary leukocytes were enzymatically dispersed and the absolute number of $\mathrm{F} 4 / 80^{+} / \mathrm{CD} 45^{+}$macrophages was quantified by flow cytometry (B). Pulmonary fungal burden data are cumulative of three experiments using five mice per time point. Results are expressed as mean $\log _{10}$ CFU per milliliter of lung homogenate \pm SEM. Flow cytometry data are cumulative results of five independent experiments using pooled leukocytes from five mice per experiment. Results are expressed as the absolute number of $\mathrm{F} 4 / 80^{+} / \mathrm{CD} 45^{+}$dual positive cells. Significant decreases were observed in C. neoformans strain $\mathrm{H} 99 \gamma$-infected compared with wild-type $C$. neoformans $\mathrm{H} 99$-infected mice. (*** $P<0.01$ ).

infection and generate protective immunity against a second infection with wild-type C. neoformans. ${ }^{23,29}$ To verify a reduction in fungal load and to assess macrophage recruitment during $C$. neoformans strain $\mathrm{H} 99 \gamma$ infection, BALB/C mice were given an intranasal inoculation with $1 \times 10^{4} \mathrm{CFU}$ of either C. neoformans strain H99 $\gamma$ or wild-type C. neoformans strain H99, and the pulmonary fungal burden was quantified on days 3,7 , and 14 postinoculation. Figure $1 \mathrm{~A}$ demonstrates that pulmonary fungal burden was significantly decreased on day 14 postinoculation in mice given an experimental pulmonary infection with $C$. neoformans strain H99 $\gamma$ compared with wild-type infected mice $(P<0.01)$. These results corroborate previous studies demonstrating that BALB/c mice given an experimental pulmonary infection with $C$. neoformans strain $\mathrm{H} 99 \gamma$ are able to completely resolve the fungal infection. ${ }^{23,29,30}$

Pulmonary leukocytes were isolated from the lungs of infected BALB/c mice on days 3,7 , and 14 postinoculation with C. neoformans strains $\mathrm{H} 99$ or $\mathrm{H} 99 \gamma$ and analyzed by flow cytometry for macrophage infiltration. We observed no significant difference in the absolute number of $\mathrm{F} 4 / 80^{+}$cells (macrophages) during infection of mice with C. neoformans strain H99 $\gamma$ compared with wild-type infected mice (Figure 1B), consistent with previous studies using 10-fold higher inocula of $C$. neoformans strains H99 or H99 $\gamma .{ }^{23}$ Thus, pulmonary infection with C. neoformans strain $\mathrm{H} 99 \gamma$ results in improved control of pulmonary C. neoformans growth that occurs without an increase in macrophage accumulation in infected lungs.

\section{Outcome of IFN- $\gamma$ Transgene Expression by C. neoformans Strain H99 $\gamma$ on Macrophage Activation in Mice During Pulmonary C. neoformans Infection}

Mice given an experimental pulmonary infection with $C$. neoformans strain $\mathrm{H} 99 \gamma$ were able to control the microbe without an increase in macrophage recruitment (Figure 1). We then investigated whether potential differences in macrophage activation phenotype account for the differ- 
Day 3
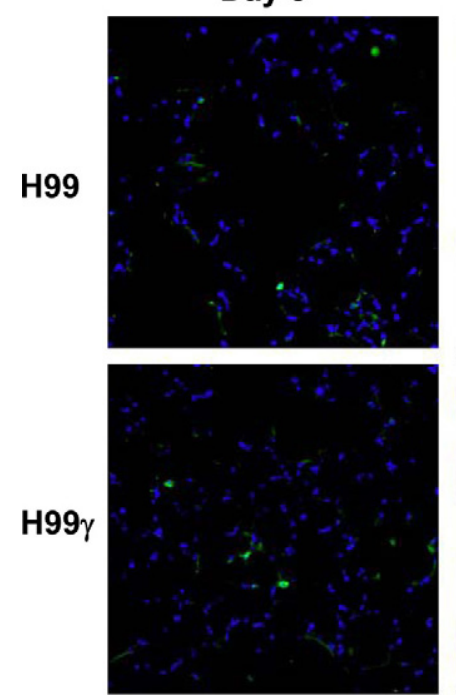

Day 7
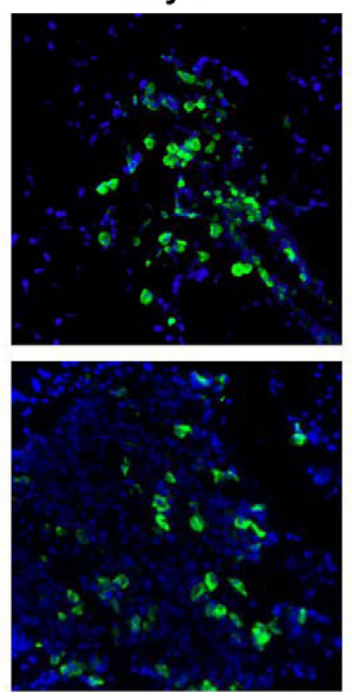
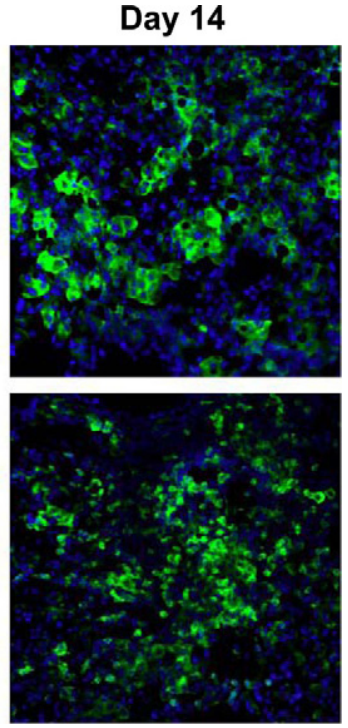

Figure 2. Mice given an experimental pulmonary infection with C. neoformans strain $\mathrm{H} 99 \gamma$ or wild-type cryptococci have comparable macrophage recruitment into the lungs during infection. BALB/c mice were given an intranasal inoculum of $1 \times 10^{4} \mathrm{CFU}$ of $C$. neoformans strains $\mathrm{H} 99$ or $\mathrm{H} 99 \gamma$ in $50 \mu \mathrm{l}$ of sterile PBS. Lungs were excised on days 3,7 , and 14 postinoculation and immediately frozen in OCT medium. Lungs were cryosectioned, and macrophage infiltration was evaluated using immunofluorescence staining with anti-F4/80 antibodies. Nuclei were counterstained with DAPI. Data shown are representative lung sections from three independent experiments (three mice per group and per experiment). Digital photographs show representative areas of lungs: $\times 20$ objective. ential response. Lungs were excised from mice given an intranasal inoculation with C. neoformans strains H99 or H99 $\gamma$ on days 3, 7, and 14 postinoculation and evaluated for hallmarks of aaMac and classically activated macrophage (caMac) by immunohistological examination. Tissue sections were immunofluorescently labeled for F4/ $80^{+}$cells (macrophages) or markers of classical (iNOS) and alternative (Arg1, CD206, and Ym1) macrophage activation. No difference in F4/80 staining was observed in the lungs of mice infected with wild-type H99 cryptococci or C. neoformans strain $\mathrm{H} 99 \gamma$ at any time point evaluated (Figure 2), a finding that corroborates previous results using flow cytometry (Figure 1B). Staining for iNOS, a marker of caMac, appeared strongest on day 7 postinoculation in mice infected with $C$. neoformans strain H99 (Figure 3), consistent with the beginning of clearance of fungi that we observed between days 7 and 14 (Figure 1A). A decline in iNOS staining (Figure 3) appeared consistent with the decline in fungal burden observed in C. neoformans strain H99 $\gamma$-infected animals on day 14 postinoculation (Figure 1A). In contrast, iNOS protein expression was not detected at any time point evaluated during infection with wild-type C. neoformans (Figure 3), despite progressive yeast growth. The aaMac hallmark proteins, Arg1, CD206, and Ym1, were expressed at low levels on days 3 and 7 postinoculation in tissues derived from mice infected with either $C$. neoformans strain $\mathrm{H} 99$ y or wild-type yeast (Figures 4, 5, and 6, for Arg1, CD206, and Ym1, respectively). However, their expression increased markedly in wild-type C. neoformans strain H99-infected lungs on day 14 postinoculation (Figures 4, 5, and 6, for Arg-1, CD206, and Ym1, respectively). In contrast, lungs from $C$. neoformans strain $\mathrm{H} 99 \gamma$-infected mice showed only very modest aaMac fluorescence on day 14 postinoculation. Thus, pulmonary infection with wild-type C. neoformans resulted in the progressive induction of aaMac proteins that was not observed in mice challenged with $C$. neoformans strain H99 $\gamma$. Furthermore, experimental pulmonary infection with $\mathrm{C}$. neoformans strain $\mathrm{H} 99 \gamma$ resulted
Day 3

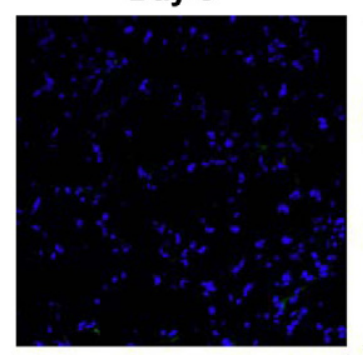

H99

$\mathrm{H} 9 \mathrm{~g}_{\gamma}$

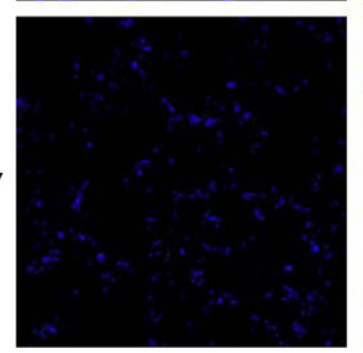

Day 7
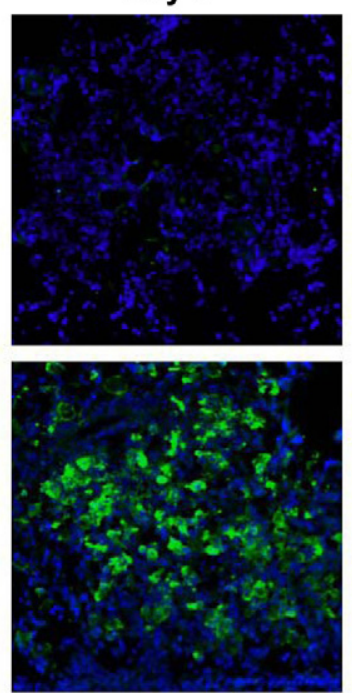

Day 14
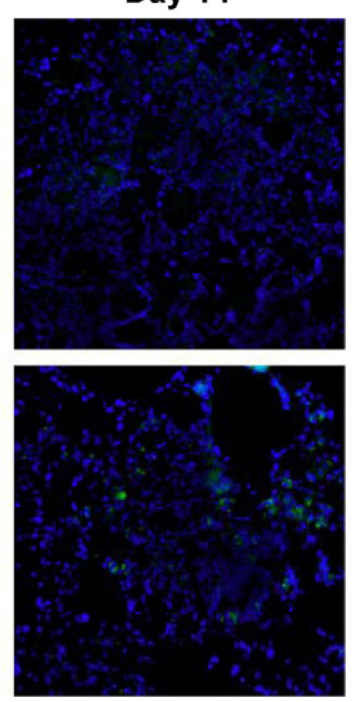

Figure 3. Pulmonary infection with $C$. neofor mans strain $\mathrm{H} 99 \gamma$, but not wild-type $C$. neofor mans, results in increased iNOS expression by pulmonary macrophages. BALB/c mice were given an intranasal inoculum of $1 \times 10^{4} \mathrm{CFU}$ of C. neoformans strains $\mathrm{H} 99$ or H99 $\gamma$ in $50 \mu$ l of sterile PBS. Lungs were excised on days 3, 7, and 14 postinoculation and immediately frozen in OCT medium. Lungs were cryosectioned, and iNOS was evaluated using immunofluorescence staining with anti-iNOS antibodies. Nuclei were counterstained with DAPI. Data shown are representative lung sections from three independent experiments (three mice per group and per experiment). Digital photographs show representative areas of lungs: $\times 20$ objective. 
Day 3

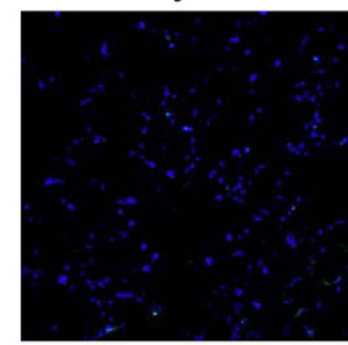

H99

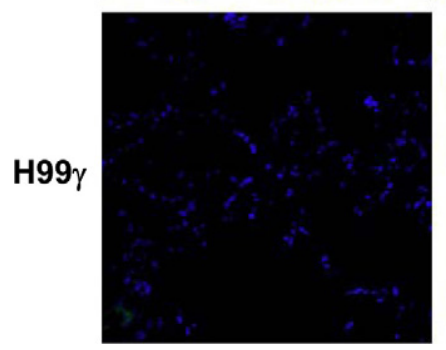

Day 7
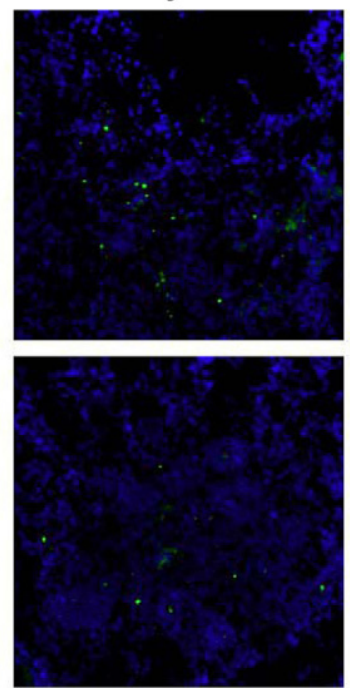

in the promotion of iNOS protein expression, a caMac hallmark.

\section{Result of IFN- $\gamma$ Transgene Expression by C. neoformans Strain H99y on Macrophage Activation and Cytokine Gene Expression in Pulmonary Macrophage-Enriched Cells}

To directly examine macrophage activation phenotype, we quantified relative mRNA expression levels for aaMac and caMac genes in pulmonary macrophage-enriched cell populations obtained from C. neoformans strain H99or $\mathrm{H} 99$-infected lungs. Pulmonary leukocytes were isolated from enzymatically dispersed lungs at days 7 and 14 after inoculation with either wild-type cryptococci or $C$. neoformans strain $\mathrm{H} 99 \gamma$. We subsequently extracted total RNA from CD11b ${ }^{+}$cell populations and evaluated macrophage activation using real-time PCR (Figure 7 ). We observed significantly increased transcript levels of
Day 14
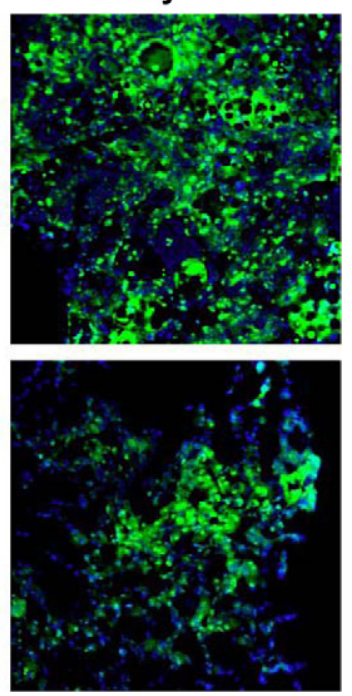

Figure 4. Mice given an experimental pulmonary infection with $C$. neoformans show increased expression of Arg1 on lung macrophages that is not observed in C. neoformans strain $\mathrm{H} 99 \gamma$-infected mice. BALB/c mice were given an intranasal inoculum of $1 \times 10^{4} \mathrm{CFU}$ of C. neoformans strains $\mathrm{H} 99$ or $\mathrm{H} 99 \gamma$ in $50 \mu \mathrm{l}$ of sterile PBS. Lungs were excised on days 3, 7, and 14 postinoculation and immediately frozen in OCT medium. Lungs were subsequently cryosectioned, and Arg1 expression was evaluated using immunofluorescence staining with antiArg1 antibodies. Nuclei were counterstained with DAPI. Data shown are representative lung sections from three independent experiments (three mice per group and per experiment). Digital photographs show representative areas of lungs: $\times 20$ objective.
Day 3

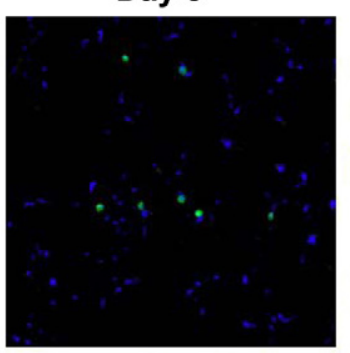

H99

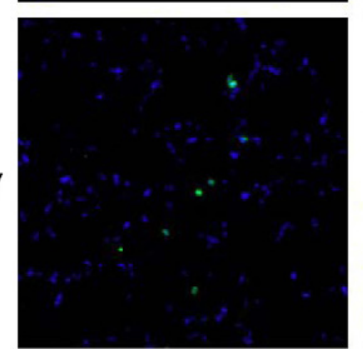

Day 7
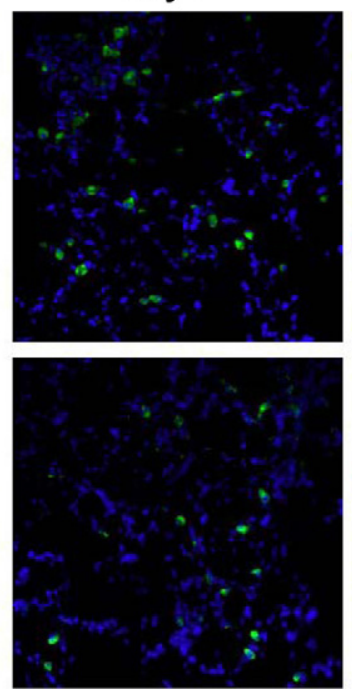

FIZZ1, Arg1, IL-4, and IL-13 (aaMac markers) on day 14 after inoculation with C. neoformans strain H99 compared with those in C. neoformans strain $\mathrm{H} 99 \gamma$-infected mice $(P<0.05,0.001,0.001$, and 0.05 for FIZZ1, Arg1, IL-4, and IL-13, respectively). In contrast, iNOS transcript expression (a marker for caMac) was significantly higher in C. neoformans strain $\mathrm{H} 99 \gamma$-infected mice on day 7 postinoculation $(P<0.05)$ compared with that in wild-type infected mice. We observed increased expression of IL-17 transcripts in mice infected with $C$ neoformans strain H99 $\gamma$ on day 14 postinoculation compared with that in wild-type infected mice; however, the increase was not statistically significant $(P<0.058)$. Thus, the macrophage gene expression profile indicates that $C$. neoformans strain $\mathrm{H} 99$ infection resulted in the differentiation of aaMac and that transgenic IFN- $\gamma$ expression during infection with $\mathrm{C}$. neoformans strain $\mathrm{H} 99 \gamma$ opposed this process, promoting caMac polarization.

Our real-time PCR studies indicate that macrophages in the lungs of $C$. neoformans-infected mice express the

\section{Day 14}
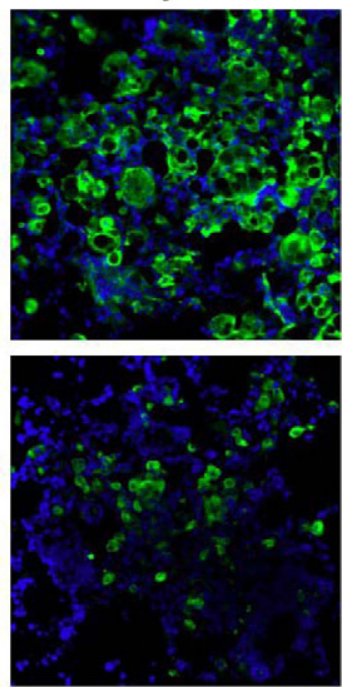

Figure 5. Pulmonary infection of mice with wild-type C. neoformans strain $\mathrm{H} 99$, but not $C$. neoformans strain $\mathrm{H} 99 \gamma$, results in increased CD206 expression on lung macrophages. $\mathrm{BALB} / \mathrm{c}$ mice were given an intranasal inoculum of $1 \times 10^{4} \mathrm{CFU}$ of $C$. neoformans strains $\mathrm{H} 99$ or $\mathrm{H} 99 \gamma$ in $50 \mu \mathrm{l}$ of sterile PBS. The lungs were excised on days 3,7 , and 14 postinoculation and immediately frozen in OCT medium. Lungs were subsequently cryosectioned, and CD206 expression was evaluated using immunofluorescence staining with anti-CD206 antibodies. Nuclei were counterstained with DAPI. Data shown are representative lung sections from three independent experiments (three mice per group and experiment). Digital photographs show representative areas of lungs: $\times 20$ objective. 


\section{Day 3}
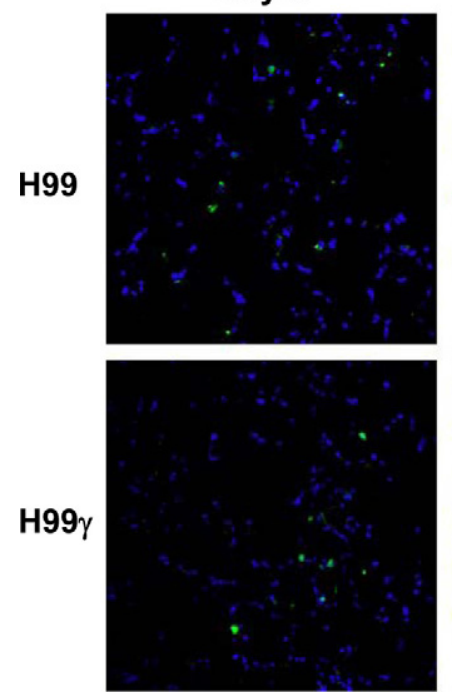

Day 7
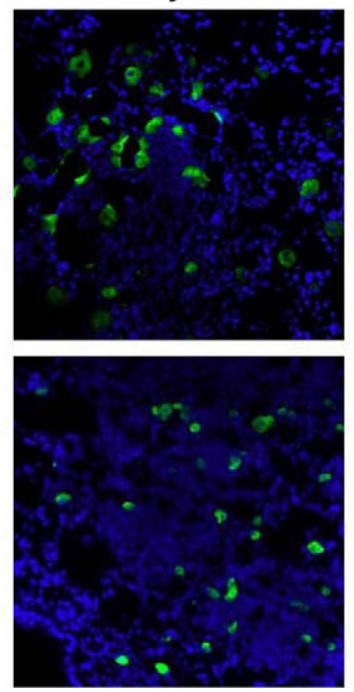

Th2-type cytokines IL-4 and IL-13. Recent studies have demonstrated that macrophages can serve as a source of $\mathrm{IL}-4$ and IL-13 production, ${ }^{31-37}$ suggesting that our findings are not unusual. Nonetheless, we sought to confirm that macrophages isolated from the lungs of mice given an experimental pulmonary C. neoformans infection express IL-4 and IL-13. Flow cytometry analysis demonstrated that $\mathrm{F} 4 / 80^{+}$cells in the lungs of mice infected with C. neoformans strain $\mathrm{H} 99 \gamma$ or wild-type cryptococci were positive for IL-4 and IL-13 on days 7 and 14 postinoculation (Figure 8). Results from three independent experiments showed no significant differences between groups (data not shown).

\section{Effect of IFN- $\gamma$ Transgene Expression by C. neoformans Strain H99y on Pulmonary Pathology}

Our data demonstrate a significant effect of IFN- $\gamma$ expression by $\mathrm{C}$. neoformans strain $\mathrm{H} 99 \gamma$ on microbial clearance

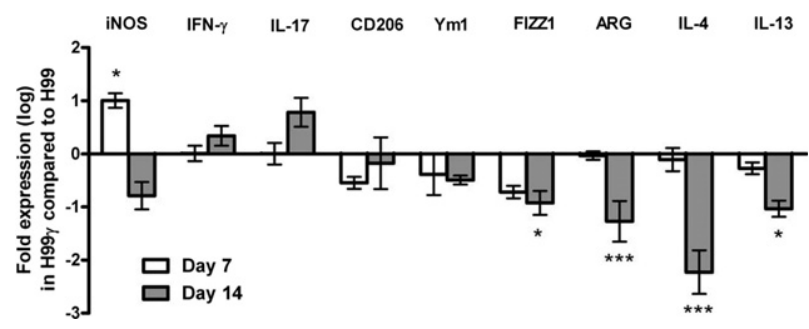

Figure 7. Real-time PCR analysis of macrophage-enriched populations show enhanced expression of aaMac and caMac transcripts in C. neoformans strains H99- and $\mathrm{H} 99 \gamma$-infected mice, respectively. BALB/c mice were given an intranasal inoculation with $1 \times 10^{4} \mathrm{CFU}$ of C. neoformans strain $\mathrm{H} 99$ or $\mathrm{H} 99 \gamma$ in $50 \mu \mathrm{l}$ of sterile PBS. Pulmonary leukocytes were isolated by enzymatic digestion on days 7 and 14 postinoculation, and macrophages were enriched for by positive selection of $\mathrm{CD}_{11} \mathrm{~b}^{+}$cells. Real-time PCR analyses of total mRNA from macrophage populations were conducted for iNOS, IFN- $\gamma$, CD206, Ym1, FIZZ1, ARG, IL-4, IL-13, IL-17, and GAPDH. Bars represent the $\log _{10}$ fold change in gene expression during infection with $C$. neoformans strain $\mathrm{H} 99 \gamma$ compared with infection with wild-type C. neoformans strain H99. Data shown are cumulative from three independent experiments using pooled leukocytes of five mice per group per experiment. Significant differences were observed at ${ }^{*} P<0.05 ;{ }^{* * * * *} P<0.001$.

\section{Day 14}
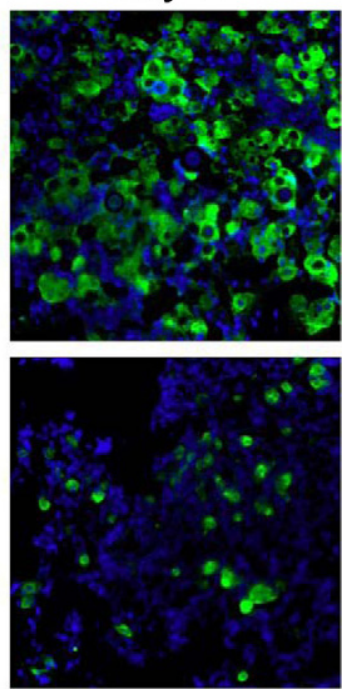

Figure 6. Pulmonary $\mathrm{Ym} 1$ expression is increased in mice after intranasal infection with wild-type $C$. neoformans but is abrogated in $C$. neoformans strain $\mathrm{H} 99 \gamma$-infected mice. BALB/C mice were given an intranasal inoculum of $1 \times$ $10^{4} \mathrm{CFU}$ of C. neoformans strains $\mathrm{H} 99$ or $\mathrm{H} 99 \gamma$ in $50 \mu \mathrm{l}$ of sterile PBS. Lungs were excised on days 3,7 , and 14 postinoculation and immediately frozen in OCT medium. Lungs were subsequently cryosectioned, and the level of $\mathrm{Ym} 1$ expression was evaluated using immunofluorescence staining with anti-Ym1 antibodies. Nuclei were counterstained with DAPI. Data shown are representative lung sections from three independent experiments (three mice per group and per experiment). Digital photographs show representative areas of lungs: $\times 20$ objective. and the expression pattern of macrophage activation markers. Therefore, we sought to determine whether mice challenged with C. neoformans strain H99 $\gamma$ experienced the severe lung pathology typically associated with wild-type C. neoformans strain H99 infection. ${ }^{12}$ Uninfected and infected lungs were collected on day 21 after infection with wild-type C. neoformans strain H99 or transgenic C. neoformans strain $\mathrm{H} 99 \gamma$ and processed for histological analysis. Sections were stained with H\&E and analyzed by light microscopy (Figure 9, A-F). Our data show that BALB/c mice infected with wild-type cryptococci developed the severe lung pathology associated with a nonprotective Th2-type cytokine response (Figure 9, B-D). C. neoformans growth was observed to be widespread, replacing most of the pulmonary airspaces (compare Figure 9A with Figure 9, B and C). Many C. neoformans organisms were also found to be unaccompanied by an inflammatory response. However, cryptococcal growth was also observed within infected macrophages (Figure 9C) consistent with the aaMac phenotype demonstrated by immunohistochemical analysis during $C$. neoformans strain H99 infection. Macrophages harbored multiple C. neoformans cells with some showing evidence of proliferation. We detected Ym1 crystals in some of the wild-type-infected macrophages along with eosinophil infiltrates (Figure 9C), suggesting a prolonged presence of Th2-type responses and aaMac in the lungs. In contrast with the development of severe pulmonary allergic mycosis in wild-type C. neoformans-infected mice, we observed a significant resolution of infection in the lungs of mice challenged with C. neoformans strain $\mathrm{H} 99 \gamma$ (Figure 9, E-F). Sections from day 21 of $C$. neoformans strain H99 $\gamma$ infection showed that a majority of the lung was clear of inflammation with few remaining mononuclear infiltrates (Figure 9E). These infiltrates present as bronchovascular neo-lymphoid tissue clusters accompanied by groups of small macrophages, as described previously in Th1-type cytokine responses, ${ }^{13}$ and occasional giant cells. Extracellular cryptococci were absent and 


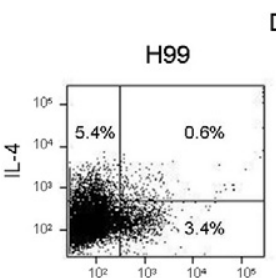

Day 7
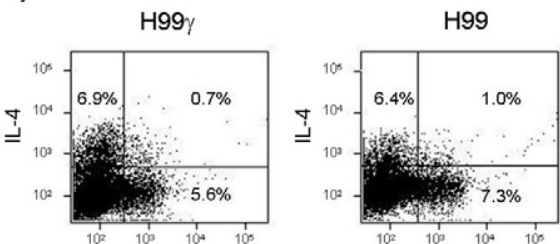

Day 14
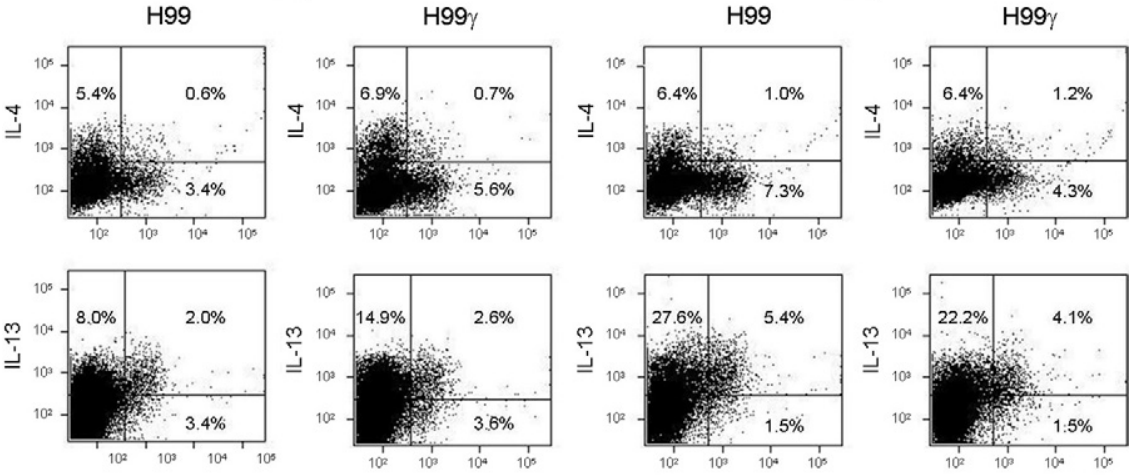

$\mathrm{F} 4 / 80$

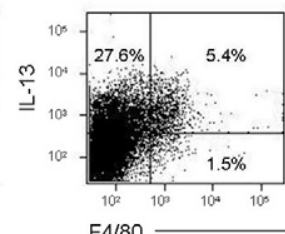

$\mathrm{F} 4 / 80$
Figure 8. Lung macrophages are positive for IL-4 and IL-13 protein expression during pulmonary infection with $C$. neoformans. BALB/c mice received an intranasal inoculum of $1 \times 10^{4} \mathrm{CFU}$ of C. neoformans strains $\mathrm{H} 99$ or $\mathrm{H} 99 \gamma$ in $50 \mu \mathrm{l}$ of sterile PBS. The lungs from each group of mice were excised at days 7 and 14 after secondary inoculation, and a single cell suspension was generated using enzymatic digestion. The leukocytes were stained with anti-mouse $\mathrm{F} 4 / 80^{+}$antibodies, fixed, permeabilized, and incubated with anti-mouse antibodies specific for IL- 4 and IL-13 and quantified by flow cytometry. Flow cytometry data are representative results of three independent experiments using pooled leukocytes from five mice per experiment. Results shown are the percentage of leukocytes expressing the indicated surface markers. only a few intracellular yeast cells were found. Furthermore, the macrophages contained small inclusion bodies weakly stained with mucicarmine, suggesting that they were the remains of ingested or destroyed cryptococci (Figure 9F). Thus, infection with C. neoformans H99 $\gamma$ is characterized not only by rapid clearance of the microbe but also by rapid resolution of inflammation, in contrast to the pathology that develops in the lungs of mice challenged with wild-type C. neoformans strain H99.

\section{Impact of IFN- $\gamma$ Transgene Expression by C. neoformans Strain H99y on Pulmonary Cytokine Production}

The local cytokine milieu appears to be critical in determining macrophage activation. ${ }^{19}$ Our final goal was to determine whether changes in the pulmonary cytokine milieu resulting from infection with $C$. neoformans strain
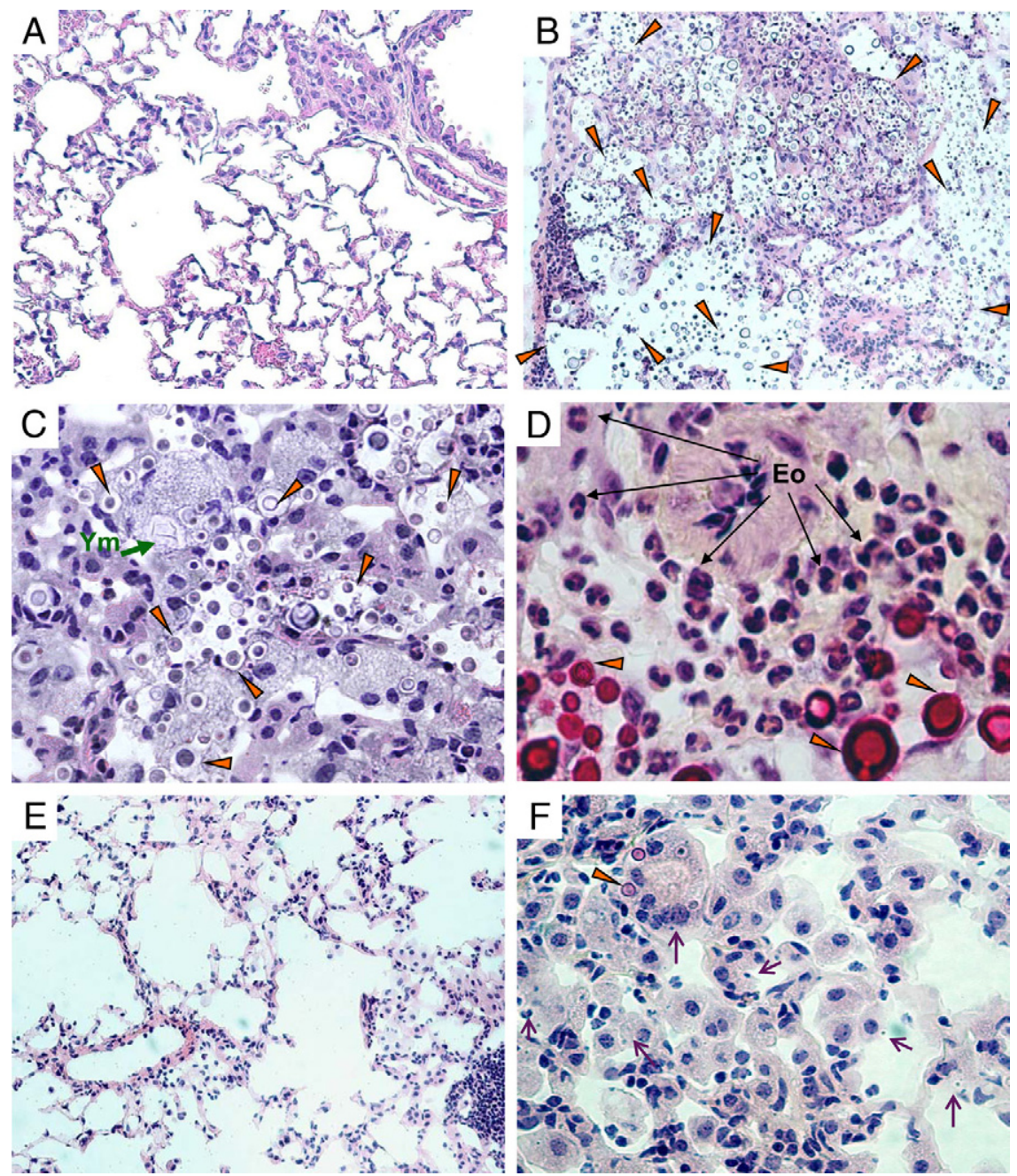

Figure 9. Pulmonary infection with $C$. neoformans $\mathrm{H} 99 \gamma$ results in rapid clearance of the cryptococci and rapid resolution of inflammation, whereas the lungs of mice challenged with wild-type C. neoformans strain $\mathrm{H} 99$ show exacerbated disease and inflammation. Lungs were collected on day 21 postinoculation from uninfected mice and mice inoculated with C. neofor mans strains $\mathrm{H} 99$ or $\mathrm{H} 99 \gamma$, frozen in OCT medium, and processed for histological analysis. Tissue sections were stained with H\&E and mucicarmine and examined under a light microscope. Digital photographs show representative areas of lungs from uninfected mice $(\times 10$ objective, $\mathbf{A}), C$. neoformans strain H99-infected mice $(\times 10, \times 40$, and $\times 100$ objectives, $\mathbf{B}-\mathbf{D})$, and $C$ neoformans $\mathrm{H} 99 \gamma$-infected mice $(\times 10$ and $\times 40$ objectives, $\mathbf{E}$ and $\mathbf{F}$ ). Note widespread intra- and extracellular cryptococci (orange arrowheads, B-D), extended macrophages with proliferating cryptococci and $\mathrm{Ym} 1 / \mathrm{Ym} 2$ crystal formation (green arrow $[\mathrm{Ym}], \mathbf{C})$, and eosinophilic infiltrate (black arrows [Eo], D) in C. neoformans strain H99-infected lungs. Note limited inflammatory response (E), few internalized yeast (orange arrowheads, F), and small inclusion bodies (purple arrows, F) consistent with elements of destroyed fungi within the macrophages in C. neoformans strain $\mathrm{H} 99 \gamma$-infected lungs. 
A

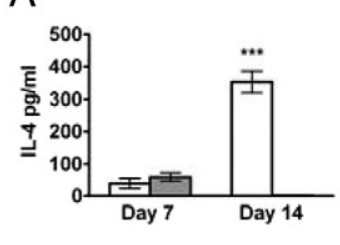

D E

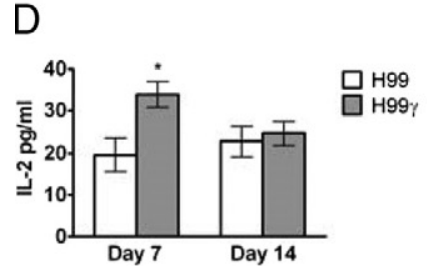

E

B

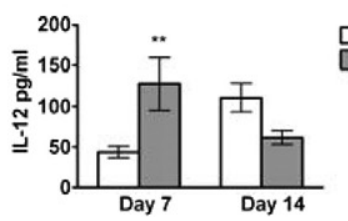

C

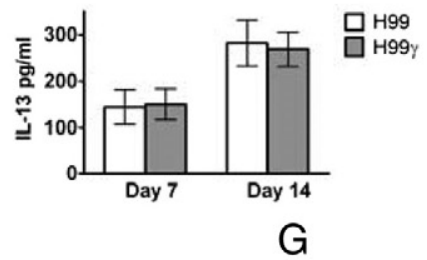

Figure 10. Pulmonary infection with C. neoformans strain H99 $\gamma$ results in the generation of a predominantly Th1-type and IL-17 cytokine environment in the lungs. BALB/c mice were given an intranasal inoculation with $1 \times 10^{4} \mathrm{CFU}$ of $C$. neoformans strain H99 or H99 $\gamma$ in $50 \mu$ l of sterile PBS. Lung homogenates were prepared from lungs excised on days 7 and 14 postinoculation and assayed for IL-4 (A), IL-5 (B), IL-13 (C), IL-2 (D), IL-12 (E), IL-17 (F), and IFN- $\gamma(\mathbf{G})$ cytokine production. Data are cumulative of two experiments using five mice each. Significant differences were observed at ${ }^{*} P<0.05 ;{ }^{* *} P<0.01 ;{ }^{*} * * * * 0.001$.

H99 $\gamma$ could be accountable for changes in macrophage phenotype. We therefore evaluated Th2-type (IL-4, IL-5, and IL-13), Th1-type (IL-2, IL-12, and IFN- $\gamma$ ), and IL-17 cytokine production in total lung homogenates derived from mice infected with wild-type C. neoformans strain H99 or transgenic C. neoformans strain H99y on days 7 and 14 postinoculation. We observed a significant elevation of the Th2-type cytokines IL-4 and IL-5 (Figure 10, A and $B$ ) on day 14 postinoculation in lungs infected with $C$. neoformans strain $\mathrm{H} 99$ ( $P<0.001$ for both), a timing that is consistent with the development of a Th2-type cytokine response $^{12}$ and with the strong aaMac polarization shown above (Figures 4-7). In contrast, IL-4 and IL-5 production was abrogated in lungs infected with $C$. neoformans strain H99 (Figure 10, A and B). Next, we observed a significant increase in the Th1-type cytokines IL-2 and IL-12 (Figure 10, D and E) and the Th17-type cytokine IL-17 (Figure 10F) on day 7 postinoculation in lungs from $C$. neoformans strain $\mathrm{H} 99 \gamma$-infected mice compared with those obtained from mice infected with wildtype yeast $(P<0.05, P<0.01$, and $P<0.001$ for IL-2, $\mathrm{IL}-12$, and $\mathrm{IL}-17$, respectively). The timing of $\mathrm{IL}-2, \mathrm{IL}-12$, and $\mathrm{IL}-17$ responses is consistent with the peak of caMac-associated iNOS expression detected in C. neoformans strain H99y-infected lungs as described above (Figures 3 and 7). Interestingly, we did not observe any significant differences in the presence of the Th2-type cytokine IL-13 (Figure 10C) or the Th1-type cytokine IFN- $\gamma$ (Figure 10G) in lung homogenates derived from wild-type C. neoformans strain H99-infected compared with $\mathrm{H} 99 \gamma$-infected mice, suggesting that these cytokines were not affected in the total environmental milieu. Altogether, these observations indicate that experimental pulmonary infection with C. neoformans strain $\mathrm{H} 99 \gamma$ resulted in the generation of a predominant Th1-type cytokine environment that was refractory to the development of Th2-type cytokine responses and aaMac polarization.

\section{Discussion}

Previous work in our laboratory has demonstrated that mice given an experimental pulmonary infection with an
IFN- $\gamma$-producing C. neoformans strain, $\mathrm{H} 99 \gamma$, have levels of pulmonary macrophage infiltrates comparable to those of mice infected with wild-type yeast, yet proceed to resolve the infection. ${ }^{23}$ In the present studies we characterized the activation phenotype of macrophages elicited during pulmonary infection with $C$. neoformans strain $\mathrm{H} 99 \gamma$ to elucidate a potential mechanism for the protection developed in these mice. We found that infection with C. neoformans strain H99 $\gamma$ was associated with increased expression of the caMac-associated gene iNOS, augmented pulmonary Th1-type cytokine production, rapid resolution of the inflammatory response, and protection from the severe lung pathology that develops in the lungs of mice infected with wild-type $C$. neoformans. Furthermore, these studies indicate that the phenotype of infiltrating macrophages is a more important determinant of protection against $C$. neoformans infection than macrophage quantity.

Indeed, Arora et $\mathrm{al}^{4}$ demonstrated that experimental pulmonary C. neoformans infection of IFN- $\gamma$ knockout mice resulted in significantly greater macrophage recruitment compared with that in wild-type mice. However, the infected IFN- $\gamma$ knockout mice displayed Th2-type polarized responses, aaMac differentiation, and reduced fungistasis, leading to progressive cryptococcal infection. Thus, it seems that augmented macrophage recruitment during experimental pulmonary C. neoformans infection is not sufficient to induce protective anti-cryptococcal immune responses. Given that pulmonary challenge with $C$. neoformans strain $\mathrm{H} 99 \gamma$ in mice results in the development of a protective Th1-type cytokine response that is conducive to caMac activation, ${ }^{19,23}$ we hypothesized that caMac are induced during pulmonary challenge with $C$. neoformans strain $\mathrm{H} 99 \gamma$.

In the present study, mice infected with C. neoformans strain H99 $\gamma$ or wild-type yeast developed disparate macrophage activation phenotypes. A robust, progressive increase in hallmark markers of aaMac differentiation (namely Arg1, CD206, FIZZ1, and Ym1) was observed during infection with the wild-type strain of C. neoformans, but these markers were repressed during infection with the transgenic C. neoformans strain H99 $\gamma$. FIZZ1 expres- 
sion is enhanced in alveolar epithelial and type II cells during allergic pulmonary inflammation and Ym1 crystallizes in alveolar spaces and in hyperactive lung macrophages and giant cells. ${ }^{17,38-41}$ Arg1 competes with iNOS for its substrate, arginine, thus depleting the cell of nitric oxide, an important intracellular fungicide. Up-regulated CD206 expression increases phagocytosis but is accompanied by decreased intracellular microbicidal activity. ${ }^{18-20,42}$ Consistent with these observations, we noted Ym1 crystal formation and $C$. neoformans yeasts displaying evidence of proliferation within macrophages of wild-type C. neoformans strain H99-infected lungs. Conversely, although we observed abrogated aaMac development during infection with $C$. neoformans strain H99 $\gamma$, we also found increased iNOS expression, an important marker of caMac. An increase in iNOS expression by caMac results in improved fungicidal activity due to the generation of reactive oxygen and nitrogen species. ${ }^{16,43-45}$ Furthermore, pulmonary macrophages from mice infected with $C$. neoformans strain H99 $\gamma$ harbored few intracellular cryptococci and contained vacuoles stained with mucicarmine, indicating cryptococci in latter stages of dissolution.

Polarized cytokine environments are perhaps the most influential determinants of macrophage activation phenotype. ${ }^{15}$ Mice challenged with wild-type C. neoformans developed a predominantly pulmonary Th2-type cytokine environment consisting of IL-4 and IL-5; conversely, pulmonary infection with transgenic $C$. neoformans strain $\mathrm{H} 99 \gamma$ resulted in a predominantly Th1-type and IL-17 cytokine environment consisting of IL-2, IL-12, and IL-17. Thus, the overall cytokine milieu generated in the lungs of mice infected with wild-type C. neoformans promotes aaMac differentiation, whereas the pulmonary cytokine environment in mice challenged with $C$. neoformans strain H99 $\gamma$ favors caMac development. Interestingly, our studies showed the presence of IL-4 and IL-13 protein expression within lung macrophages of mice after pulmonary C. neoformans infection. These results corroborate several other recent studies, indicating that macrophages can serve as a source of IL-4 and IL-13. ${ }^{31-37}$ Also intriguing, IFN- $\gamma$ protein levels were not significantly different in pulmonary homogenates of mice challenged with C. neoformans strain H99y, despite observation of significant increases in other Th1-type cytokines compared with those in wild-type-infected mice. Previous studies in mice administered a 10-fold higher inoculum of C. neoformans strain $\mathrm{H} 99 \mathrm{y}$ than that used in the current study showed significantly increased pulmonary expression of IFN- $\gamma$ and IL-17 compared with that of wild-typeinfected mice. ${ }^{23}$ Recent studies have shown that the proliferation of $C$. neoformans within macrophages and cryptococcal expulsion rates are significantly lower after treatment with Th1-type cytokines and IL-17. ${ }^{46}$ Alternatively, IL-4 and IL-13 treatment significantly increased intracellular yeast proliferation. Thus, it appears that the inoculum used in this study was able to induce a Th1type and IL-17 polarized environment that was not predominated by IFN- $\gamma$. The presence of a significant biological effect indicates that production of IFN- $\gamma$ by the microbe is sufficient to evoke protection without altering the overall IFN- $\gamma$ production by leukocytes. It is likely that the close interaction between C. neoformans strain H99 and macrophages during phagocytosis is sufficient to induce the essential effects of IFN- $\gamma$ production, such as major histocompatibility class II expression, ${ }^{10,11,47}$ enhanced macrophage respiratory burst, and the release of proinflammatory cytokines by macrophages. ${ }^{18,19,42}$ These responses are expected to augment macrophage activity toward the resolution of cryptococcal infection. In addition, the transgenic strain is likely to have induced other cellular responses that may influence caMac differentiation.

The question remains whether IFN- $\gamma$ alone is sufficient to induce caMac differentiation in mice challenged with C. neoformans strain H99 $\gamma$. High IL-4/13 environments induce aaMac marker expression but are antagonistic to the development of caMac. ${ }^{15}$ Specifically, IL-4 and IL-13, acting through a common receptor chain (IL-4R $\alpha$ ), enhance the expression of CD206, FIZZ1, Ym1, and Arg1 while suppressing macrophage IFN- $\gamma$ production and subsequent iNOS-mediated nitric oxide production. ${ }^{15,39,41,48}$ In contrast, IFN- $\gamma$ down-regulates CD206 expression and enhances the expression of iNOS that, in turn, enhances nitric oxide synthesis. ${ }^{16,18,19,42,49}$ Indeed, C. neoformans-infected IFN- $\gamma$ knockout mice generate aaMac and experience progressive infection. ${ }^{4}$ In contrast, previous reports using IL13-deficient mice clearly showed an abrogation of aaMac induction that correlated with increased resistance to pulmonary C. neoformans infection. ${ }^{11}$ Likewise, the results herein demonstrate a preclusion of aaMac induction in mice after infection with C. neoformans strain H99 $\gamma$. However, the present study is the first report to show the generation of caMac and sterilizing immunity, in vivo, during experimental pulmonary $C$. neoformans infection. We were able to demonstrate that pulmonary infection with $C$. neoformans strain $\mathrm{H} 99 \gamma$ was resolved in mice and resulted in the induction of a predominant Th1-type and IL-17 cytokine environment in the lungs and caMac differentiation, coupled with the abrogation of Th2-type cytokine production and aaMac. Therefore, it seems that not only is the prevention of aaMac differentiation essential in the protective response to $C$. neoformans but also that caMac are induced.

Altogether, the results show that pulmonary infection with C. neoformans strain $\mathrm{H} 99 \mathrm{y}$ in mice results in the induction of Th1-type and IL-17 cytokine responses, classical macrophage activation, and the resolution of infection. Pulmonary infection of mice with wild-type cryptococci induced Th2type polarized responses, alternative macrophage activation, pulmonary eosinophilia, and progressive disease associated with disease in accordance with previous independent studies. ${ }^{4,11}$ Our studies suggest that therapies targeting the induction of classical macrophage activation have the potential to generate protection against pulmonary cryptococcosis in human patients.

\section{Acknowledgments}

We thank Jose Lopez-Ribot, Pharm.D., Ph.D., Sarah Bubeck, Ph.D., and Judy Teale, Ph.D., for critical reading of the manuscript. 


\section{References}

1. Mitchell TG, Perfect JR: Cryptococcosis in the era of AIDS-100 years after the discovery of Cryptococcus neoformans. Clin Microbiol Rev 1995, 8:515-548

2. Powderly WG: Cryptococcal meningitis and AIDS. Clin Infect Dis 1993, 17:837-842

3. Aguirre K, Havell EA, Gibson GW, Johnson LL: Role of tumor necrosis factor and $\gamma$ interferon in acquired resistance to Cryptococcus neoformans in the central nervous system of mice. Infect Immun 1995 63:1725-1731

4. Arora S, Hernandez Y, Erb-Downward JR, McDonald RA, Toews GB, Huffnagle GB: Role of IFN- $\gamma$ in regulating T2 immunity and the development of alternatively activated macrophages during allergic bronchopulmonary mycosis. J Immunol 2005, 174:6346-6356

5. Collins HL, Bancroft GJ: Cytokine enhancement of complement-dependent phagocytosis by macrophages: synergy of tumor necrosis factor- $\alpha$ and granulocyte-macrophage colony-stimulating factor for phagocytosis of Cryptococcus neoformans. Eur J Immunol 1992, 22:1447-1454

6. Flesch IE, Schwamberger G, Kaufmann SH: Fungicidal activity of IFN- $\gamma$-activated macrophages. Extracellular killing of Cryptococcus neoformans. J Immunol 1989, 142:3219-3224

7. Huffnagle GB, Lipscomb MF: Cells and cytokines in pulmonary cryptococcosis. Res Immunol 1998, 149:387-396; discussion 512-514

8. Levitz SM, DiBenedetto DJ: Differential stimulation of murine resident peritoneal cells by selectively opsonized encapsulated and acapsular Cryptococcus neoformans. Infect Immun 1988, 56:2544-2551

9. Loke P, Nair MG, Parkinson J, Guiliano D, Blaxter M, Allen JE: IL-4 dependent alternatively-activated macrophages have a distinctive in vivo gene expression phenotype. BMC Immunol 2002, 3:7

10. Mody CH, Tyler CL, Sitrin RG, Jackson C, Toews GB: Interferon- $\gamma$ activates rat alveolar macrophages for anticryptococcal activity. Am J Respir Cell Mol Biol 1991, 5:19-26

11. Müller U, Stenzel W, Kohler G, Werner C, Polte T, Hansen G, Schutze $N$, Straubinger RK, Blessing M, McKenzie AN, Brombacher F, Alber G: IL-13 induces disease-promoting type 2 cytokines, alternatively activated macrophages and allergic inflammation during pulmonary infection of mice with Cryptococcus neoformans. J Immunol 2007, 179:5367-5377

12. Osterholzer JJ, Surana R, Milam JE, Montano GT, Chen GH, Sonstein J, Curtis JL, Huffnagle GB, Toews GB, Olszewski MA: Cryptococcal urease promotes the accumulation of immature dendritic cells and a non-protective T2 immune response within the lung. Am J Pathol 2009, 174:932-943

13. Chen GH, Olszewski MA, McDonald RA, Wells JC, Paine R 3rd, Huffnagle GB, Toews GB: Role of granulocyte macrophage colonystimulating factor in host defense against pulmonary Cryptococcus neoformans infection during murine allergic bronchopulmonary mycosis. Am J Pathol 2007, 170:1028-1040

14. Ito T, Schaller M, Hogaboam CM, Standiford TJ, Chensue SW, Kunkel SL: TLR9 activation is a key event for the maintenance of a mycobacterial antigen-elicited pulmonary granulomatous response. Eur J Immunol 2007, 37:2847-2855

15. Martinez FO, Helming L, Gordon S: Alternative activation of macrophages: an immunologic functional perspective. Annu Rev Immunol 2009, 27:451-483

16. Hesse M, Modolell M, La Flamme AC, Schito M, Fuentes JM, Cheever AW, Pearce EJ, Wynn TA: Differential regulation of nitric oxide synthase- 2 and arginase- 1 by type 1/type 2 cytokines in vivo: granulomatous pathology is shaped by the pattern of L-arginine metabolism. J Immunol 2001, 167:6533-6544

17. Chang NC, Hung SI, Hwa KY, Kato I, Chen JE, Liu CH, Chang AC: A macrophage protein. Ym1, transiently expressed during inflammation is a novel mammalian lectin. J Biol Chem 2001, 276:17497-17506

18. Goerdt S, Politz O, Schledzewski K, Birk R, Gratchev A, Guillot P Hakiy N, Klemke CD, Dippel E, Kodelja V, Orfanos CE: Alternative versus classical activation of macrophages. Pathobiology 1999 67:222-226

19. Gordon S: Alternative activation of macrophages. Nat Rev Immunol 2003, 3:23-35

20. Stein M, Keshav S, Harris N, Gordon S: Interleukin 4 potently enhances murine macrophage mannose receptor activity: a marker of alternative immunologic macrophage activation. J Exp Med 1992 176:287-292

21. De Groot PW, Ram AF, Klis FM: Features and functions of covalently linked proteins in fungal cell walls. Fungal Genet Biol 2005, 42:657-675

22. Noverr MC, Cox GM, Perfect JR, Huffnagle GB: Role of PLB1 in pulmonary inflammation and cryptococcal eicosanoid production. Infect Immun 2003, 71:1538-1547

23. Wormley FL Jr, Perfect JR, Steele C, Cox GM: Protection against cryptococcosis by using a murine $\gamma$ interferon-producing Cryptococcus neoformans strain. Infect Immun 2007, 75:1453-1462

24. Cox GM, McDade HC, Chen SC, Tucker SC, Gottfredsson M, Wright LC, Sorrell TC, Leidich SD, Casadevall A, Ghannoum MA, Perfect JR: Extracellular phospholipase activity is a virulence factor for Cryptococcus neoformans. Mol Microbiol 2001, 39:166-175

25. Cox GM, Mukherjee J, Cole GT, Casadevall A, Perfect JR: Urease as a virulence factor in experimental cryptococcosis. Infect Immun 2000, 68:443-448

26. Aarskog NK, Vedeler CA: Real-time quantitative polymerase chain reaction. A new method that detects both the peripheral myelin protein 22 duplication in Charcot-Marie-Tooth type $1 \mathrm{~A}$ disease and the peripheral myelin protein 22 deletion in hereditary neuropathy with liability to pressure palsies. Hum Genet 2000, 107:494-498

27. Chang JT, Chen IH, Liao CT, Wang HM, Hsu YM, Hung KF, Lin CJ, Hsieh LL, Cheng AJ: A reverse transcription comparative real-time PCR method for quantitative detection of angiogenic growth factors in head and neck cancer patients. Clin Biochem 2002, 35:591-596

28. Vandesompele J, De Preter K, Pattyn F, Poppe B, Van Roy N, De Paepe A, Speleman F: Accurate normalization of real-time quantitative RT-PCR data by geometric averaging of multiple internal control genes. Genome Biol 2002, 3:RESEARCH0034

29. Young M, Macias S, Thomas D, Wormley FL Jr: A proteomic-based approach for the identification of immunodominant Cryptococcus neoformans proteins. Proteomics 2009, 9:2578-2588

30. Wozniak KL, Ravi S, Macias S, Young ML, Olszewski MA, Steele C, Wormley FL: Insights into the mechanisms of protective immunity against Cryptococcus neoformans infection using a mouse model of pulmonary cryptococcosis. PLoS One 2009, 4:e6854

31. Büttner C, Skupin A, Reimann T, Rieber EP, Unteregger G, Geyer P, Frank KH: Local production of interleukin-4 during radiation-induced pneumonitis and pulmonary fibrosis in rats: macrophages as a prominent source of interleukin-4. Am J Respir Cell Mol Biol 1997, 17: 315-325

32. Hancock A, Armstrong L, Gama R, Millar A: Production of interleukin 13 by alveolar macrophages from normal and fibrotic lung. Am $J$ Respir Cell Mol Biol 1998, 18:60-65

33. Hauber HP, Gholami D, Meyer A, Pforte A: Increased interleukin-13 expression in patients with sarcoidosis. Thorax 2003, 58:519-524

34. Pouliot P, Turmel V, Gelinas E, Laviolette M, Bissonnette EY: Interleukin-4 production by human alveolar macrophages. Clin Exp Allergy 2005, 35:804-810

35. Prieto J, Lensmar C, Roquet A, van der Ploeg I, Gigliotti D, Eklund A Grunewald J: Increased interleukin-13 mRNA expression in bronchoalveolar lavage cells of atopic patients with mild asthma after repeated low-dose allergen provocations. Respir Med 2000, 94: $806-814$

36. Shirey KA, Cole LE, Keegan AD, Vogel SN: Francisella tularensis live vaccine strain induces macrophage alternative activation as a survival mechanism. J Immunol 2008, 181:4159-4167

37. Szymczak WA, Deepe GS Jr: The CCL7-CCL2-CCR2 axis regulates IL-4 production in lungs and fungal immunity. J Immunol 2009, 183: $1964-1974$

38. Guo L, Johnson RS, Schuh JC: Biochemical characterization of endogenously formed eosinophilic crystals in the lungs of mice. J Biol Chem 2000, 275:8032-8037

39. Loke P, MacDonald AS, Robb A, Maizels RM, Allen JE: Alternatively activated macrophages induced by nematode infection inhibit proliferation via cell-to-cell contact. Eur J Immunol 2000, 30:2669-2678

40. Raes G, De Baetselier P, Noel W, Beschin A, Brombacher F, Hassanzadeh Gh G: Differential expression of FIZZ1 and Ym1 in alternatively versus classically activated macrophages. J Leukoc Biol 2002, 71:597-602

41. Welch JS, Escoubet-Lozach L, Sykes DB, Liddiard K, Greaves DR, Glass CK: TH2 cytokines and allergic challenge induce Ym1 expres- 
sion in macrophages by a STAT6-dependent mechanism. J Biol Chem 2002, 277:42821-42829

42. Allen JE, Loke P: Divergent roles for macrophages in lymphatic filariasis. Parasite Immunol 2001, 23:345-352

43. Forman HJ, Torres M: Reactive oxygen species and cell signaling: respiratory burst in macrophage signaling. Am $\mathrm{J}$ Respir Crit Care Med 2002, 166:S4-S8

44. Hickman-Davis JM, Fang FC, Nathan C, Shepherd VL, Voelker DR, Wright JR: Lung surfactant and reactive oxygen-nitrogen species: antimicrobial activity and host-pathogen interactions. Am J Physiol Lung Cell Mol Physiol 2001, 281:L517-L523

45. Hickman-Davis JM, O'Reilly P, Davis IC, Peti-Peterdi J, Davis G, Young KR, Devlin RB, Matalon S: Killing of Klebsiella pneumoniae by human alveolar macrophages. Am J Physiol Lung Cell Mol Physiol 2002, 282:L944-L956

46. Voelz K, Lammas DA, May RC: Cytokine signaling regulates the outcome of intracellular macrophage parasitism by Cryptococcus neoformans. Infect Immun 2009, 77:3450-3457

47. Noël W, Raes G, Hassanzadeh Ghassabeh G, De Baetselier P, Beschin A: Alternatively activated macrophages during parasite infections. Trends Parasitol 2004, 20:126-133

48. Schindler H, Lutz MB, Rollinghoff M, Bogdan C: The production of IFN- $\gamma$ by IL-12/IL-18-activated macrophages requires STAT4 signaling and is inhibited by IL-4. J Immunol 2001, 166:3075-3082

49. Aderem A, Ulevitch RJ: Toll-like receptors in the induction of the innate immune response. Nature 2000, 406:782-787 